\title{
The Effect of Daily Low Dose Tadalafil on Cerebral Perfusion and Cognition in Patients with Erectile Dysfunction and Mild Cognitive Impairment
}

\author{
Jin Bong Choi ${ }^{1}$, Kang Jun $\mathrm{Cho}^{1}$, Joon Chul Kim ${ }^{1}$, Chung Ho Kim${ }^{2}$, Yong-An Chung ${ }^{3}$, Hyeonseok S. Jeong ${ }^{3}$, \\ Yong Soo Shim ${ }^{4}$, Jun Sung Koh ${ }^{1}$ \\ Departments of ${ }^{1}$ Urology, ${ }^{2}$ Nuclear Medicine, and ${ }^{4}$ Neurology, Bucheon St. Mary's Hospital, College of Medicine, The Catholic University \\ of Korea, ${ }^{3}$ Department of Radiology, Incheon St. Mary's Hospital, College of Medicine, The Catholic University of Korea, Seoul, Korea
}

\begin{abstract}
Objective: The aims of this study were to investigate the effects of daily low-dose tadalafil on cognitive function and to examine whether there was a change in cerebral blood flow (CBF) in patients with erectile dysfunction (ED) and mild cognitive impairment.

Methods: Male patients aged 50 to 75 years with at least three months of ED (International Index of Erectile Function [IIEF]-5 score $\leq 21$ ) and mild cognitive impairment (Montreal Cognitive Assessment [MoCA] score $\leq 22$ ) were included in the study. The subjects were prescribed a low-dose PDE5 inhibitor (tadalafil $5 \mathrm{mg}$ ) to be taken once daily for eight weeks. Changes in MoCA score and single-photon emission computed tomography (SPECT) study between the two time-points were assessed by paired $t$ tests.

Results: Overall, 30 male patients were assigned to the treatment group in this study and 25 patients completed the eight-week treatment course. Five patients were withdrawn due to adverse events such as myalgia and dizziness. Mean baseline IIEF and MoCA scores were $7.52 \pm 4.84$ and $18.92 \pm 1.78$. After the eight-week treatment, mean IIEF and MoCA scores were increased to $12.92 \pm 7.27(p<0.05)$ and $21.8 \pm 1.71(p<0.05)$, respectively. Patients showed increased relative regional CBF in the postcentral gyrus, precuneus, and brainstem after tadalafil administration versus at baseline $(p<0.001)$.

Conclusion: The results of this prospective clinical study suggest that daily use of tadalafil $5 \mathrm{mg}$ increases some regional CBF and improves cognitive function in patients with $\mathrm{ED}$ and mild cognitive impairment.
\end{abstract}

KEY WORDS: Cerebrovascular circulation; Cognition; Phosphodiesterase inhibitors.

\section{INTRODUCTION}

Phosphodiesterase (PDE) 5 inhibitors regulate signaling pathways by elevating cyclic guanosine monophosphate (cGMP) levels. They may furthermore enhance cerebral blood flow (CBF) through the vasoactive activity of CGMP. ${ }^{1)}$ A decline in CBF has been suggested to be associated with cognitive dysfunction. ${ }^{2)}$

Except for blood flow, several other mechanisms that

Received: November 27, 2018 / Revised: December 14, 2018 Accepted: December 18, 2018

Address for correspondence: Jun Sung Koh

Department of Urology, Bucheon St. Mary's Hospital, College of Medicine, Catholic University of Korea, 327 Sosa-ro, Wonmi-gu, Bucheon 14647, Korea

E-mail: gostraight@catholic.ac.kr

ORCID: https://orcid.org/0000-0001-7432-4209 could explain the cognition-enhancing effects of PDE5 inhibitors have been suggested to date. Some reports describe an emotional arousal effect of PDE5 inhibitors and suggest that cognitive function could be improved as a consequence of emotional arousal. ${ }^{3,4)}$ Enhanced second messenger signaling also results in the facilitation of longterm potentiation (LTP) processes. ${ }^{5)}$ Hippocampal LTP is considered to represent a pivotal synaptic model for learning and memory consolidation. ${ }^{6)}$ As such, there has been an increasing interest in the development of PDE5 inhibitors for cognition enhancement.

Tadalafil is a PDE5 inhibitor that has been used to treat erectile dysfunction (ED). An increasing number of patients have been treated with a once-daily low dose of tadalafil since it was approved by the United States Food

(ㄷ) This is an Open-Access article distributed under the terms of the Creative Commons Attribution Non-Commercial License (http://creativecommons.org/licenses/by-nc/4.0) which permits unrestricted non-commercial use, distribution, and reproduction in any medium, provided the original work is properly cited. 
and Drug Administration for use in cases of benign prostate hyperplasia. Tadalafil can cross the blood-brain barrier and has been reported to improve cognitive function in animal models. ${ }^{7)}$ However, few studies on the effects of tadalafil on cognition have been completed to date in humans, though there have been a number of papers published that have investigated the effects of sildenafil on human cognition. ${ }^{8,9)}$ In addition, ED is associated with poor cognitive performance, especially in the aspects of attention, executive functioning, and processing speed, because vascular problems are the most common etiology of ED. ${ }^{10)}$ Patients with ED are also at an increased risk for dementia later in life. ${ }^{11)}$

Considering these concepts about PDE5 inhibitors, the aims of this study were to investigate the effects of daily low-dose tadalafil on cognitive function and to examine whether there was a change in cerebral perfusion in patients with ED and mild cognitive impairment.

\section{METHODS}

\section{Study Design}

This prospective clinical study was conducted at single medical center in Korea. The study was performed in accordance with the ethical principles of the Declaration of Helsinki. Written informed consent was received from each patient prior to their participation in the study. This study was approved by the Institutional Review Board of the Catholic University of Korea (HC17MISI0012).

The subjects were prescribed a low-dose PDE5 inhibitor (tadalafil $5 \mathrm{mg}$ ) once daily for eight weeks. Subjects were instructed to take one tablet about two hours prior to sexual intercourse or before bedtime. Assessments were completed at eight weeks after the study initiation, including single-photon emission computed tomography (SPECT) study, which was performed both at baseline and at eight weeks after. Patients reported adverse events at each visit. Incidence, type, and severity of adverse event were reported. A physical examination and vital sign assessment were performed for analysis at the beginning of the study and at four weeks and eight weeks.

\section{Subjects}

Male patients aged 50 years to 75 years with at least three months of ED (International Index of Erectile Function [IIEF]-5 score $\leq 21$ ) and mild cognitive impairment
(Montreal Cognitive Assessment [MoCA] score $\leq 22$ ) were included in the study. MoCA is one of the representative tools for assessing general cognitive function. ${ }^{12)}$ Exclusion criteria included serious cerebrovascular or cardiovascular conditions within the previous six months; uncontrolled hypertension and hypotension; uncontrolled diabetes and arrhythmia; major psychiatric disorder or neurological disorder; history of glaucoma; history of major hematological, renal, or hepatic abnormalities; and/or taking nitrate or nitric oxide donors. Patients currently taking tricyclic antidepressants, monoamine oxidase inhibitors, or selective serotonin reuptake inhibitors as well as those with a contraindication for using tadalafil were also excluded. The use of cytochrome P450 3A4 inhibitors and drugs for the treatment of dementia (e.g., donepezil, rivastigmine, galantamine, or memantine) were discontinued during the treatment period.

There have been a few studies on the effects of PDE5 inhibitors on cerebral perfusion conducted to date. In addition, the present study was conducted as a means of preliminary research for potential application in clinical studies involving a larger number of subjects in the future. Therefore, we decided that 30 subjects were needed considering a beta error 0.15 and dropout rate of $10 \%$ at a significance level of $0.05 .^{13)}$

\section{Image Analysis}

A SPECT study was performed twice in each patient using a dual-head, variable-angle SPECT gamma camera (Infinia Hawkeye; GE Healthcare, Haifa, Israel). The baseline study was performed at 15 minutes after intravenous injection of Tc-99m-hexamethylpropylenaminoxime, while the second SPECT study was performed at eight weeks after the same. ${ }^{14)}$ SPECT data were analyzed using Statistical Parametric Mapping 12 (SPM; Wellcome Department of Cognitive Neurology, Institute of Neurology, London, UK). All images were spatially normalized to the SPM SPECT template (Montreal Neurological Institute, McGill University, Montreal, Canada) using a 12-parameter affine transformation, followed by nonlinear transformations and trilinear interpolation. Images were resliced with a voxel size of $2 \times 2 \times 2 \mathrm{~mm}$ and smoothed with a 12-mm, full-width half-maximum Gaussian kernel. Normalized regional CBF maps were created using proportional scaling. Global counts were scaled to $50 \mathrm{ml} / 100 \mathrm{~g} / \mathrm{min}$. 


\section{Statistical Analysis}

Changes in MoCA score between the two time-points (before and after the administration of eight weeks of tadalafil) were assessed by paired $t$ tests. A voxel-wise paired $t$ test was conducted to evaluate regional perfusion changes between the baseline and follow-up. The height threshold was $p<0.05$ and the extent threshold was 100 or more contiguous voxels, respectively. Changes in regional CBF values were extracted from each significant cluster using the MarsBar toolbox (http://marsbar.sourceforge. net/).

\section{RESULTS}

\section{Subjects}

Overall, 30 male patients were assigned to the treatment group in this study and 25 of them completed the eight-week treatment course. One patient withdrew consent and four patients were withdrawn from the study due to adverse events such as myalgia, dizziness, and abdominal pain. Efficacy analyses were performed in the per-protocol population. The demographic data and baseline characteristics of subjects are shown in Table 1 . The average age of the 25 patients was 64.36 years \pm 5.82 years and the mean duration of ED was 30.12 months \pm 17.78 months. The mean baseline IIEF and MoCA scores were

Table 1. Demographic and baseline parameters

\begin{tabular}{lc}
\hline \multicolumn{1}{c}{ Parameter } & Patient $(\mathrm{n}=25)$ \\
\hline Age $(\mathrm{yr})$ & $64.36 \pm 5.82$ \\
Body mass index $\left(\mathrm{kg} / \mathrm{m}^{2}\right)$ & $25.44 \pm 2.33$ \\
Cause of ED & 7 \\
Psychogenic & 18 \\
Organic & $30.12 \pm 17.78$ \\
Duration of ED $(\mathrm{mo})$ & \\
Past medical history & 22 \\
BPH & 9 \\
Diabetes & 12 \\
Hypertension & 10 \\
Hyperlipidemia & 2 \\
Depression & 0 \\
Obscessive disorder & 20 \\
Other disease & $7.52 \pm 4.84$ \\
IIEF-5 scroe & $18.92 \pm 1.78$ \\
MoCA score &
\end{tabular}

Values are presented as mean \pm standard deviation or number only. $\mathrm{ED}$, erectile dysfunction; $\mathrm{BPH}$, benign prostate hyperplasia; IIEF, International Index of Erectile Function; MoCA, Montreal Cognitive Assessment.
$7.52 \pm 4.84$ and $18.92 \pm 1.78$, respectively.

\section{Primary Efficacy Variable}

Changes in regional CBF are shown in Table 2. Regional CBF was significantly increased in the postcentral gyrus ( $\mathrm{T}$ score $=4.34, p<0.001$ ), precuneus (T score $=4.21, p<$ 0.001 ), and brainstem ( $\mathrm{T}$ score $=4.06, p<0.001$ ) following the administration of eight weeks of tadalafil. However, there was a decrease in blood flow in the hippocampus as compared with the baseline hippocampal blood flow. Figure 1 also shows changes in regional CBF at eight weeks. The red-yellow color indicates the increase in the follow-up versus at baseline, while the blue-green color indicates a decrease in the follow-up versus at baseline.

\section{Secondary Efficacy Variables}

The IIEF and MoCA scores at eight weeks were $12.92 \pm$ 7.27 and $21.80 \pm 1.71$. Mean changes of the IIEF and MoCA scores were $5.40 \pm 6.52$ and $2.88 \pm 1.61$. Analyses of mean changes of the MoCA scores revealed that the cognitive functions of the subjects were significantly improved after administration of eight weeks of tadalafil ( $p<$ 0.001). Subanalysis of MoCA showed statistically significant changes in the remaining items except for "Visuospatial/Executive" and "Orientation" (Table 3).

\section{Safety and Tolerability}

Although four patients in the treatment group experienced adverse events such as myalgia, dizziness, and abdominal pain, all adverse events were mild to moderate in severity. Furthermore, most drug-related adverse events were attenuated without any treatment. There was no significant drug-related change in clinical parameters.

Table 2. Changes in regional cerebral blood flow

\begin{tabular}{lllrl}
\hline \multicolumn{1}{c}{ Region } & T score & $p$ value & $\begin{array}{r}\text { Coordinates* } \\
(\mathrm{x}, \mathrm{y}, \mathrm{z})\end{array}$ & $\begin{array}{c}\text { Cluster size } \\
(\text { voxel) }\end{array}$ \\
\hline Baseline $<$ follow-up & & & & \\
$\quad$ L postcentral gyrus & 4.34 & $<0.001$ & $-66,-12,22$ & 131 \\
$\quad$ R precuneus & 4.21 & $<0.001$ & $8,-62,22$ & 279 \\
$\quad$ Brainstem & 4.06 & $<0.001$ & $-2,-34,-2$ & 478 \\
Baseline $>$ follow-up & & & & \\
$\quad$ R hippocampus & 5.00 & $<0.001$ & $20,-12,-22$ & 327 \\
\hline
\end{tabular}

*The coordinates refer to the Montreal Neurological Institute coordinate system. 


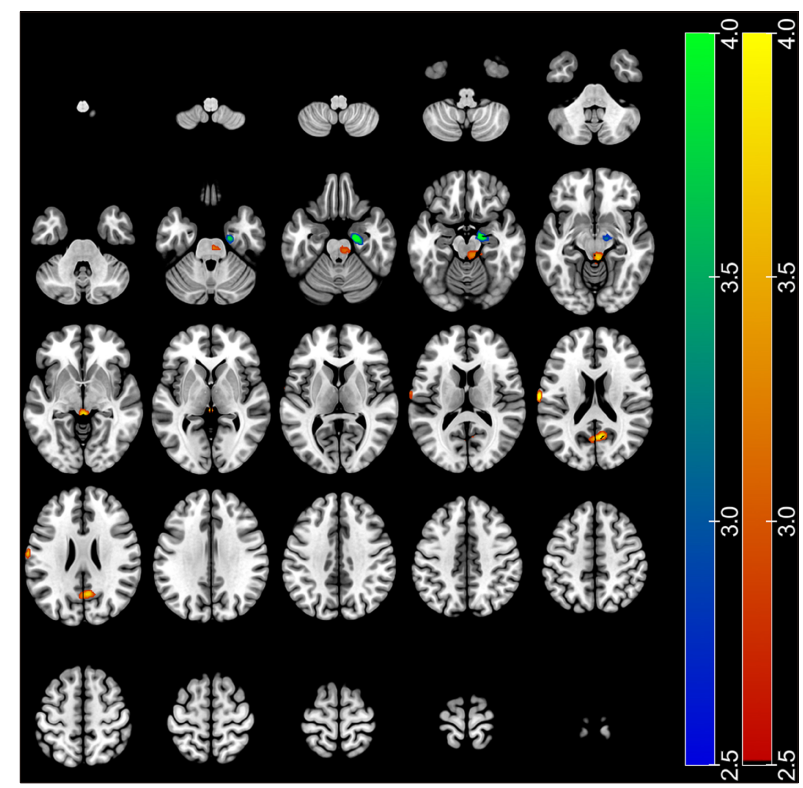

Fig. 1. Changes in regional cerebral blood flow at follow-up. At each voxel, increases or decreases in brain perfusion are indicated by a red-yellow or blue-green color, respectively. Images are shown in neurological convention. The color bars represent voxel-level $t$ values.

\section{DISCUSSION}

The main findings of this prospective study are: (1) daily low-dose tadalafil administration increased relative regional CBF in the postcentral gyrus, precuneus, and brainstem and (2) that cognitive function was significantly improved after the administration of tadalafil for eight weeks.

There are several mechanisms of action that could explain the cognition-enhancing effects of PDE5 inhibitors. ${ }^{5)}$ Among these mechanisms, increases in blood flow and glucose metabolism have been numerously reported that might be related to the cognitive enhancements seen after PDE5 inhibitor treatments in animal models. Localized CBF was increased after seven days of oral administration of sildenafil at a dose of $2 \mathrm{mg} / \mathrm{kg}$ upon measuring relative CBF velocity with laser Doppler flowmetry in anesthetized rats. ${ }^{15)}$ The administration of sildenafil in rats with embolic stroke enhanced angiogenesis and selectively increased the CBF in the ischemic lesion. ${ }^{16)}$ Tadalafil treatment also increased cerebral vascular density and endothelial cells around the ischemic boundary in a rat model of embolic stroke. ${ }^{17)}$

Several studies have reported CBF changes after the administration of PDE5 inhibitors in human, though there has remained some controversy regarding the subject. In
Table 3. Mean change between assessmentsat baseline and eight weeks

\begin{tabular}{|c|c|c|c|}
\hline Parameter & Visit & Mean scrore $(n=25)$ & $p$ value* \\
\hline \multirow[t]{3}{*}{ IIEF } & Baseline & $7.52 \pm 4.84$ & $<0.001$ \\
\hline & Week 8 & $12.92 \pm 7.27$ & \\
\hline & Mean change & $5.40 \pm 6.52$ & \\
\hline \multirow[t]{3}{*}{ MoCA } & Baseline & $18.92 \pm 1.78$ & $<0.001$ \\
\hline & Week 8 & $21.80 \pm 1.71$ & \\
\hline & Mean change & $2.88 \pm 1.61$ & \\
\hline \multirow[t]{2}{*}{ Visuospatial/Executive } & Baseline & $3.08 \pm 1.22$ & 1.00 \\
\hline & Week 8 & $3.08 \pm 0.81$ & \\
\hline \multirow[t]{2}{*}{ Naming } & Baseline & $2.40 \pm 0.71$ & 0.005 \\
\hline & Week 8 & $2.68 \pm 0.63$ & \\
\hline \multirow[t]{2}{*}{ Attention } & Baseline & $3.32 \pm 0.95$ & $<0.001$ \\
\hline & Week 8 & $4.44 \pm 1.08$ & \\
\hline \multirow[t]{2}{*}{ Language } & Baseline & $1.88 \pm 0.73$ & 0.005 \\
\hline & Week 8 & $2.44 \pm 0.65$ & \\
\hline \multirow[t]{2}{*}{ Abstraction } & Baseline & $0.52 \pm 0.51$ & 0.005 \\
\hline & Week 8 & $0.81 \pm 0.41$ & \\
\hline \multirow[t]{2}{*}{ Delayed recall } & Baseline & $1.92 \pm 0.91$ & 0.018 \\
\hline & Week 8 & $2.36 \pm 0.76$ & \\
\hline \multirow[t]{2}{*}{ Orientation } & Baseline & $5.80 \pm 0.58$ & 0.096 \\
\hline & Week 8 & $0.60 \pm 0.00$ & \\
\hline
\end{tabular}

Values are presented as mean \pm standard deviation.

IIEF, International Index of Erectile Function; MoCA, Montreal Cognitive Assessment.

${ }^{*} p$ values between the baseline and week 8

one study, there was a significant increase of cerebral oxygenated hemoglobin and total hemoglobin concentration after intravenous sildenafil administration in children with elevated pulmonary vascular resistance due to congenital heart defects after cardiac surgery. ${ }^{18)}$ Sheng et al. ${ }^{19)}$ suggested that a 50-mg single dose of sildenafil improves cerebral hemodynamic function in patients with Alzheimer's disease. Specifically, they used magnetic resonance imaging to investigate the alterations of CBF and found that increased CBF was most pronounced in the bilateral medial temporal lobes. On the contrary, other reports suggested that sildenafil has no significant effects on $\mathrm{CBF}$, which researchers measuring the regional $\mathrm{CBF}$ in the perfusion area of the middle cerebral artery using transcranial Doppler or SPECT. ${ }^{20,21)}$

Our previous clinical study revealed that daily dosing with a PDE5 inhibitor improves cognitive function, depression, and somatization using the Korean version of the Mini-Mental State Examination, though we could not provide a clear mechanism for this change at that time. ${ }^{22)}$ Therefore, we planned to determine the precise mechanisms of the cognitive-enhancing effects of PDE5 inhibitors by the measurement of CBF with comprehensive 
neuropsychometric assessments. In this study, regional CBF was measured using SPECT. Patients showed increased relative regional $\mathrm{CBF}$ in the postcentral gyrus, precuneus, and brainstem after tadalafil administration as compared with baseline, though there was a decrease in CBF in the hippocampus as compared with baseline.

Recently, it was hypothesized that the brainstem constitutes an essential part of the cerebello-cerebral neural network, which acts as a subordinate to cognitive function, though the brainstem has been known to provide motor and sensory innervation. ${ }^{23)}$ The precuneus plays a role in episodic memory, visuospatial processing, and consciousness. ${ }^{24)} \mathrm{A}$ recent positron-emission tomography analysis of patients with amnestic mild cognitive impairment showed metabolic alterations in the precuneus, though a number of previous studies using structural magnetic resonance imaging focused on the hippocampus in patients with mild cognitive impairment. ${ }^{25)}$

The present study has several limitations. First, this study is just a comparative study of cognitive function and CBF change before and after the administration of tadalafil due to an unethical issue of radiation exposure in placebo controlled group. Therefore, a randomized placebo controlled study should be needed to confirm the effectiveness of daily low-dose tadalafil on cognitive function. It is also necessary to adjust for confounding factors such as education that may affect cognitive function that were not included in this study. Second, the accurate measurement of hippocampal blood flow is difficult due to its small size, variable distribution of arteries, and location; furthermore, it can be supplied by the branches of the artery and the numerous internal hippocampal arterioles arising from these vessels, which can make for throughflow error. Therefore, SPECT alone might be insufficient to measure regional $\mathrm{CBF}$ in the hippocampus accurately. ${ }^{26,27)}$

In conclusion, the results of this prospective clinical study suggest that the daily use of tadalafil $5 \mathrm{mg}$ increases some regional CBF and improves cognitive function in patients with $\mathrm{ED}$ and mild cognitive impairment. However, a randomized controlled study using a larger sample size is required to elucidate the clear underlying mechanism(s) that might explain the cognition-enhancing effects of tadalafil. Furthermore, new imaging modalities to measure $\mathrm{CBF}$ better should be validated.

\section{Acknowledgments}

The study was funded by Hanmi Pharm Co., Ltd.

\section{- Conflicts of Interest}

No potential conflict of interest relevant to this article was reported.

\section{REFERENCES}

1. Reneerkens OA, Rutten K, Akkerman S, Blokland A, Shaffer $\mathrm{CL}$, Menniti FS, et al. Phosphodiesterase type 5 (PDE5) inhibition improves object recognition memory: indications for central and peripheral mechanisms. Neurobiol Learn Mem 2012;97:370-379.

2. Bertsch K, Hagemann D, Hermes M, Walter C, Khan R, Naumann E. Resting cerebral blood flow, attention, and aging. Brain Res 2009;1267:77-88.

3. Kurt M, Bilge SS, Aksoz E, Kukula O, Celik S, Kesim Y. Effect of sildenafil on anxiety in the plus-maze test in mice. Pol J Pharmacol 2004;56:353-357.

4. Milman HA, Arnold SB. Neurologic, psychological, and aggressive disturbances with sildenafil. Ann Pharmacother 2002; 36:1129-1134.

5. Reneerkens OA, Rutten K, Steinbusch HW, Blokland A, Prickaerts J. Selective phosphodiesterase inhibitors: a promising target for cognition enhancement. Psychopharmacology (Berl) 2009;202:419-443.

6. Bliss TV, Collingridge GL. A synaptic model of memory: long-term potentiation in the hippocampus. Nature 1993; 361:31-39.

7. García-Barroso C, Ricobaraza A, Pascual-Lucas M, Unceta N, Rico AJ, Goicolea MA, et al. Tadalafil crosses the blood-brain barrier and reverses cognitive dysfunction in a mouse model of AD. Neuropharmacology 2013;64:114-123.

8. Schultheiss D, Müller SV, Nager W, Stief CG, Schlote N, Jonas $\mathrm{U}$, et al. Central effects of sildenafil (Viagra) on auditory selective attention and verbal recognition memory in humans: a study with event-related brain potentials. World J Urol 2001;19:46-50.

9. Grass $\mathrm{H}$, Klotz T, Fathian-Sabet B, Berghaus G, Engelmann U, Käferstein H. Sildenafil (Viagra): is there an influence on psychological performance? Int Urol Nephrol 2001;32:409-412.

10. Moore CS, Grant MD, Zink TA, Panizzon MS, Franz CE, Logue $\mathrm{MW}$, et al. Erectile dysfunction, vascular risk, and cognitive performance in late middle age. Psychol Aging 2014;29:163172.

11. Yang CM, Shen YC, Weng SF, Wang JJ, Tien KJ. Increased risk of dementia in patients with erectile dysfunction: a population-based, propensity score-matched, longitudinal follow-up study. Medicine (Baltimore) 2015;94:e990.

12. Lim KB, Kim J, Lee HJ, Yoo J, You EC, Kang J. Correlation between Montreal Cognitive Assessment and functional outcome in subacute stroke patients with cognitive dysfunction. 
Ann Rehabil Med 2018;42:26-34.

13. Julious SA. Sample sizes for clinical trials with normal data. Stat Med 2004;23:1921-1986.

14. Lorberboym M, Makhline E, Lampl Y. Regional cerebral blood flow following single-dose and continuous-dose tadalafil after stroke. Acta Neurol Scand 2014;130:380-386.

15. Zhang R, Wang Y, Zhang L, Zhang Z, Tsang W, Lu M, et al. Sildenafil (Viagra) induces neurogenesis and promotes functional recovery after stroke in rats. Stroke 2002;33:2675-2680.

16. Li L, Jiang Q, Zhang L, Ding G, Gang Zhang Z, Li Q, et al. Angiogenesis and improved cerebral blood flow in the ischemic boundary area detected by MRI after administration of sildenafil to rats with embolic stroke. Brain Res 2007;1132:185192.

17. Zhang L, Zhang Z, Zhang RL, Cui Y, LaPointe MC, Silver B, et al. Tadalafil, a long-acting type 5 phosphodiesterase isoenzyme inhibitor, improves neurological functional recovery in a rat model of embolic stroke. Brain Res 2006;1118:192-198.

18. Nagdyman N, Fleck T, Bitterling B, Ewert P, Abdul-Khaliq $\mathrm{H}$, Stiller B, et al. Influence of intravenous sildenafil on cerebral oxygenation measured by near-infrared spectroscopy in infants after cardiac surgery. Pediatr Res 2006;59:462-465.

19. Sheng M, Lu H, Liu P, Li Y, Ravi H, Peng SL, et al. Sildenafil improves vascular and metabolic function in patients with Alzheimer's disease. J Alzheimers Dis 2017;60:1351-1364.

20. Arnavaz A, Aurich A, Weissenborn K, Hartmann U, Emrich $\mathrm{HM}$, Schneider U. Effect of sildenafil (Viagra) on cerebral blood flow velocity: a pilot study. Psychiatry Res 2003;122:
207-209.

21. Kruuse C, Thomsen LL, Jacobsen TB, Olesen J. The phosphodiesterase 5 inhibitor sildenafil has no effect on cerebral blood flow or blood velocity, but nevertheless induces headache in healthy subjects. J Cereb Blood Flow Metab 2002;22:11241131.

22. Shim YS, Pae CU, Cho KJ, Kim SW, Kim JC, Koh JS. Effects of daily low-dose treatment with phosphodiesterase type 5 inhibitor on cognition, depression, somatization and erectile function in patients with erectile dysfunction: a double-blind, placebo-controlled study. Int I Impot Res 2014;26:76-80.

23. D'aes T, Mariën P. Cognitive and affective disturbances fo/lowing focal brainstem lesions: a review and report of three cases. Cerebellum 2015;14:317-340.

24. Cavanna AE, Trimble MR. The precuneus: a review of its functional anatomy and behavioural correlates. Brain 2006;129: 564-583.

25. Haussmann R, Werner A, Gruschwitz A, Osterrath A, Lange J, Donix $\mathrm{KL}$, et al. Precuneus structure changes in amnestic mild cognitive impairment. Am J Alzheimers Dis Other Demen 2017:32:22-26.

26. Talati P, Rane S, Skinner J, Gore J, Heckers S. Increased hippocampal blood volume and normal blood flow in schizophrenia. Psychiatry Res 2015;232:219-225.

27. Rusinek H, Brys M, Glodzik L, Switalski R, Tsui WH, Haas F, et al. Hippocampal blood flow in normal aging measured with arterial spin labeling at 3T. Magn Reson Med 2011;65:128137. 